\title{
Polarization of a Charge Qubit Strongly Coupled to a Voltage-Driven Quantum Point Contact
}

\author{
I. Snyman ${ }^{1}$ and Y. V. Nazarov ${ }^{2}$ \\ ${ }^{1}$ Instituut-Lorentz, Universiteit Leiden, P.O. Box 9506, 2300 RA Leiden, The Netherlands \\ ${ }^{2}$ Kavli Institute of Nanoscience, Delft University of Technology, 2628 CJ Delft, The Netherlands
}

(Received 12 December 2006; published 31 August 2007)

\begin{abstract}
We study a charge qubit with level splitting $\varepsilon$, coupled to a quantum point contact driven by voltage $V$. In the limit of weak coupling, the qubit polarization shows cusps at $\varepsilon= \pm e V$. We show that, for stronger couplings, prominent peculiarities occur at fractions $\varepsilon= \pm \mathrm{eV} / 2$. Further increase of the coupling leads to a polarization corresponding to a pseudo Boltzmann distribution with an effective temperature $\sim e V$.
\end{abstract}

DOI: 10.1103/PhysRevLett.99.096802

PACS numbers: 73.23.-b, 03.67.Lx, 73.40.Jn, 85.30.Hi

The quantum point contact [1] has become a basic concept in the field of quantum transport owing to its simplicity. Its common experimental realization is a narrow constriction that connects two metallic reservoirs. An adequate theoretical description for this setup is a noninteracting one-dimensional electron gas interrupted by a potential barrier. The barrier is completely characterized by its scattering matrix. This enables the scattering approach to quantum transport [2].

Despite the correctness of the noninteracting electron description, truly many-body quantum correlations in a QPC do exist and are observable. These manifest themselves in the full counting statistics (FCS) of electron transfers [3] and allow for detection of two-particle entanglement [4] through the measurement of nonlocal current correlations. This suggests that the observation of manybody effects in a QPC crucially relies on a proper detection scheme.

In this Letter, we probe a QPC with a charge qubit. Such a device has already been realized using single and double quantum dots. Previously, the QPC has been used as a detector of the qubit state $[5,6]$. We propose a scheme in which these roles are reversed. Provided the qubit and QPC are coupled strongly, switching between the qubit states is accompanied by severe Fermi-Sea shake-up in the QPC. The ratio of switching rates determines the qubit polarization. The dc current in the QPC reads the qubit polarization. Thereby we obtain information about the Fermi-Sea shake-up in the QPC.

For our results to apply, the qubit transition rate induced by the QPC should therefore dominate the rate due to coupling with other environmental modes. We estimate this requirement to be fulfilled already in the weak coupling regime.

Before analyzing the system in detail, the following qualitative conclusions can be drawn. The qubit owes its detection capabilities to the following fact: In order to be excited it has to absorb a quantum $\varepsilon$ of energy from the QPC. Here $\varepsilon$ is the qubit level splitting, a parameter that can be tuned easily in an experiment by means of a gate voltage. The QPC supplies the energy by transferring charge from the high voltage reservoir to the low voltage reservoir. The transfer of charge $q$ allows qubit transitions for level splittings $\varepsilon<q V, V$ being the bias-voltage applied. Thus, the creation of excitations in the QPC is correlated with qubit switching.

We can assume that successive switchings of the qubit between its states $|1\rangle$ and $|2\rangle$ are rare and uncorrelated. The qubit dynamics are then characterized by the rates $\Gamma_{21}$ to switch from state $|1\rangle$ to state $|2\rangle$ and $\Gamma_{12}$ from $|2\rangle$ to $|1\rangle$. The stationary probability to find the qubit in state $|2\rangle$, or polarization for short, is determined by detailed balance to be $p_{2}=\Gamma_{21} /\left(\Gamma_{12}+\Gamma_{21}\right)$. The polarization can be observed experimentally by measuring the current in the QPC. The current displays random telegraph noise, switching between two values $I_{1}$ and $I_{2}$. These correspond to the qubit being in the state $|1\rangle$ or $|2\rangle$, respectively. The dc current $I$ gives the average over many switches and is thus related to the stationary probability by $I=$ $\left(1-p_{2}\right) I_{1}+p_{2} I_{2}$. The values of $I_{1}, I_{2}$, and $I$ are determined through measurement and $p_{2}$ is inferred.

When the QPC and qubit are weakly coupled $[7,8]$, a single electron is transferred [9]. This liberates at most energy $\mathrm{eV}$, implying that the rate $\Gamma_{21}$ is zero when $\varepsilon>\mathrm{eV}$ and the rate $\Gamma_{12}$ is zero when $\varepsilon<-e V$. The resulting $p_{2}$ changes from 1 to 0 upon increasing $\epsilon$ within the interval $-e V<\epsilon<e V$. Cusps at $\varepsilon= \pm e V$ signify that the charge $e$ is transferred. [See Fig. 2(a)].

Guided by our understanding of weak coupling we can speculate as follows about what happens at stronger couplings. Apart from single electron transfers, we also expect the coordinated transfers of groups of electrons. A group of $n$ electrons can provide up to $n e V$ of energy to the qubit. Therefore, peculiarities in $p_{2}$ should appear at the corresponding level splittings $\varepsilon= \pm n e V, n=1,2,3, \ldots$ [10] However, it is not a priori obvious that these peculiarities are pronounced enough to be observed. The reason is the decoherence of the qubit states induced by electrons passing through the QPC. This smooths out peculiarities at the energy scale that is the inverse of the decoherence time. In the strong coupling regime, especially when the qubit couples to many QPC channels, the decoherence time is 
estimated to be short so that smoothing is severe. As a result, it is not clear whether peculiarities at $n e V$ are the dominant feature at strong coupling.

Therefore, strong coupling of the QPC and the qubit requires quantitative analysis. We have reduced the problem to the evaluation of a determinant of an infinitedimensional Wiener-Hopf operator. We calculated the determinant numerically for a single channel QPC and found that peculiarities at multiples of $\mathrm{eV}$ are minute. Their contribution to $p_{2}$ does not exceed $10^{-4}$ and is seen only at logarithmic scale and at moderate couplings. Instead, far more prominent features occurs at $\varepsilon=\frac{1}{2} \mathrm{eV}$. General reasoning does not predict this. Straightforward energy balance arguments suggest that a charge $e / 2$ has been transferred between the QPC reservoirs. We are tempted to view this as a fractionally charged excitation generated by the qubit. However, the setup under consideration does not support an independent determination of the excitation charge. If we further increase the coupling, by adding channels to the QPC, we find a pseudo Boltzmann distribution $p_{2}=\left[1+\exp \left(\lambda \varepsilon / k_{B} T^{*}\right)\right]^{-1}$, with the effective temperature $k_{B} T^{*}$ of the order $\mathrm{eV}$. All peculiarities disappear due to decoherence.

Let us now turn to the details of our analysis. The system is illustrated in Fig. 1. The Hamiltonian for the system is

$$
\hat{H}=\hat{T}+\hat{U}_{1}|1\rangle\left\langle 1\left|+\left(\hat{U}_{2}+\varepsilon\right)\right| 2\right\rangle\langle 2|+\gamma(|1\rangle\langle 2|+| 2\rangle\langle 1|) .
$$

The operator $\hat{T}$ represents the kinetic energy of QPC electrons. The operator $\hat{U}_{k}$ describes the potential barrier seen by QPC electrons when the qubit is in state $k=1,2$ and corresponds to a scattering matrix $\check{s}_{k}$ in the scattering approach. (We use an inverted caret to indicate a matrix in the space of transport channels.) QPC electrons do not interact directly with each other but rather with the qubit. This interaction is the only qubit relaxation mechanism included in our model. We work in the limit $\gamma \rightarrow 0$, where the inelastic transition rates $\Gamma_{12,21}$ between qubit states are
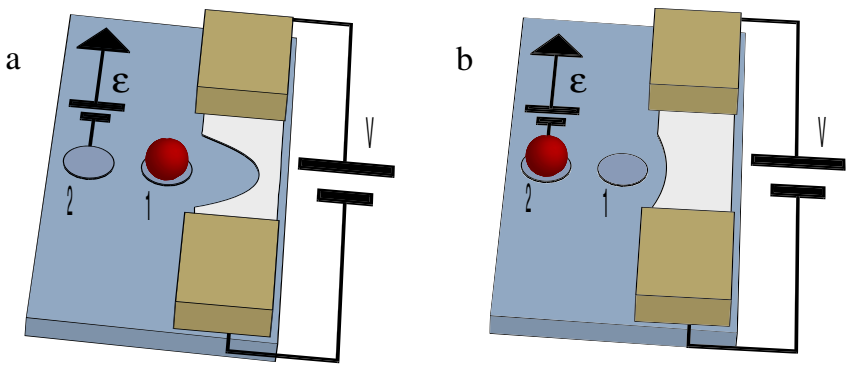

FIG. 1 (color online). A schematic picture of the system considered. It consists of a charge qubit coupled to a QPC. The shape of the QPC constriction, and hence its scattering matrix, depends on the state of the qubit. The QPC is biased at voltage $V$. A gate voltage controls the qubit level splitting $\varepsilon$. There is a small tunneling rate $\gamma$ between qubit states. small compared to the energies $e V$ and $\varepsilon$. In this case, the qubit switching events can be regarded as independent and incoherent.

Now consider the qubit transition rate $\Gamma_{21}$. To lowest order in the tunneling amplitude $\gamma$ it is given by

$$
\begin{aligned}
\Gamma_{21}= & 2 \gamma^{2} \operatorname{Re} \int_{-\infty}^{0} d \tau e^{i \varepsilon \tau} \lim _{t_{0} \rightarrow-\infty} \operatorname{tr}\left[e^{i \hat{H}_{2} \tau} e^{-i \hat{H}_{1}\left(\tau-t_{0}\right)}\right. \\
& \left.\times \rho_{0} e^{i \hat{H}_{1}\left(\tau-t_{0}\right)}\right] .
\end{aligned}
$$

This is the usual Fermi Golden Rule. The Hamiltonians $\hat{H}_{1}$ and $\hat{H}_{2}$ are given by $\hat{H}_{k}=\hat{T}+\hat{U}_{k}$ and represent QPC dynamics when the qubit is held fixed in state $|k\rangle$. The trace is over QPC states, and $\rho_{0}$ is the initial QPC density matrix.

The evaluation of the integrand is a special case of a general problem in the extended Keldysh formalism [11]. The task is to evaluate the trace of a density matrix after "bra's" have evolved with a time-dependent Hamiltonian $\hat{H}_{-}(t)$ and "kets" with a different Hamiltonian $\hat{H}_{+}(t)$.

$$
e^{\mathcal{A}}=\operatorname{tr}\left[\mathcal{T}^{+} e^{-i \int_{-\infty}^{\infty} d t \hat{H}_{+}(t)} \rho_{0} \mathcal{T}^{-} e^{i \int_{-\infty}^{\infty} d t \hat{H}_{-}(t)}\right] .
$$

We implemented the scattering approach to obtain the general formula

$$
\mathcal{A}=\operatorname{tr} \ln \left[\hat{s}_{-}(1-\hat{f})+\hat{s}_{+} \hat{f}\right]-\operatorname{tr} \ln \hat{s}_{-} .
$$

The operators $\hat{s}_{ \pm}$and $\hat{f}$ have both continuous and discrete indices. The continuous indices refer to energy, or in the Fourier transformed representation, to time. The discrete indices refer to transport channel space. The operators $\hat{s}_{ \pm}=\breve{s}_{ \pm}(t) \delta\left(t-t^{\prime}\right)$ are diagonal in time. The timedependent scattering matrices $\breve{s}_{ \pm}(t)$ describe scattering by the Hamiltonians $\hat{H}_{ \pm}(t)$ at instant $t$. (It is the hallmark of the scattering approach to express quantities in terms of scattering matrices rather than Hamiltonians.) The operator $\hat{f}=\breve{f}(E) \delta\left(E-E^{\prime}\right)$ is diagonal in the energy representation. The matrix $\breve{f}(E)$ is diagonal in channel space, representing the individual electron filling factors in the different channels. A full derivation of Eq. (4) will be given elsewhere. It generalizes similar relations published in $[12,13]$.

In order to apply the general result to Eq. (2), the timedependent scattering matrices $\breve{s}_{ \pm}(t)$ are chosen as $\check{s}_{+}(t)=$ $\check{s}_{1}+\theta(t-\tau) \theta(-t)\left(\check{s}_{2}-\check{s}_{1}\right)$ and $\check{s}_{-}=\check{s}_{1}$. The QPC scattering matrices $\check{s}_{1}\left(\breve{s}_{2}\right)$ with the qubit in the state 1(2) are the most important parameters of our approach.

Without a bias-voltage applied, the QPC-qubit setup exhibits the physics of the Anderson orthogonality catastrophe [14]. For the equilibrium QPC, the problem can be mapped [12] onto the classic Fermi edge singularity (FES) problem [15-17]. In effect the authors of [12] computed $\mathcal{A}$ in equilibrium. Our setup is simpler than the generic FES problem since there is no tunneling from the qubit to the QPC. As a result, out of all processes considered in [12], we only need the so-called closed loop diagrams. The 
relevant part of the FES result for our setup is an anomalous power law $\Gamma_{21}^{(0)}(\varepsilon)=\theta(-\varepsilon) \frac{1}{|\varepsilon|}\left(\frac{|\varepsilon|}{E_{\mathrm{c} .0 .}}\right)^{\alpha}$ for the equilibrium rate. Here $E_{\text {c.o. }}$ is an upper cutoff energy. The anomalous exponent $\alpha$ is determined by the eigenvalues of $\hat{s}_{2}^{\dagger} \hat{s}_{1}$ [18] as $\alpha=\frac{1}{4 \pi^{2}}\left|\operatorname{Tr} \ln ^{2}\left(\hat{s}_{f}^{\dagger} \hat{s}_{i}\right)\right|$. The logarithm is defined on the branch $(-\pi, \pi]$. For a one or two channel point contact, $0<\alpha<1$.

We now give the details of our calculation for the rates out of equilibrium. From Eqs. (2) and (4) it follows that $\Gamma_{21}(\varepsilon) \propto\left|\gamma^{2}\right| \int_{-\infty}^{\infty} d \tau e^{-i \varepsilon \tau} \operatorname{det} \hat{Q}^{(V)}(\tau)$. For positive times $\tau$, the operator $\hat{Q}^{(V)}(\tau)$ is defined as [12]

$$
\hat{Q}^{(V)}(\tau)=1+\left(\check{s}_{2}^{-1} \check{s}_{1}-1\right) \hat{\Pi}(\tau) \hat{f}^{(V)},
$$

while for negative $\tau, \hat{Q}^{(V)}(\tau)=\hat{Q}^{(V)}(-\tau)^{\dagger}$. The timeinterval operator $\hat{\Pi}(\tau)=\delta\left(t-t^{\prime}\right) \theta(t) \theta(\tau-t)$ is diagonal in time and acts as the identity operator in channel space for times $t=t^{\prime} \in[0, \tau]$ and as the zero-operator outside this time interval.

For the purpose of numerical calculation of the determinant we have to regularize $\hat{Q}^{(V)}(\tau)$. This is done by multiplying with the inverse of the zero-bias operator to define a new operator $\tilde{Q}(\tau)=\hat{Q}^{(0)}(\tau)^{-1} \hat{Q}^{(V)}(\tau)$. Its determinant is evaluated numerically. The rate $\Gamma_{21}(\epsilon)$ at bias voltage $V$ is then expressed as the convolution $\Gamma_{21}(\varepsilon)=$ $\int \frac{d \varepsilon^{\prime}}{2 \pi} \Gamma_{21}^{\mathrm{eq}}\left(\varepsilon-\varepsilon^{\prime}\right) \tilde{P}\left(\varepsilon^{\prime}\right)$ of the equilibrium rate and the Fourier transform of $\tilde{P}(\tau)=\operatorname{det} \tilde{Q}^{(V)}(\tau)$, that contains all effects of the bias-voltage $V$.

We implemented this calculation numerically, and computed the probability $p_{2}$ to find the qubit in state $|2\rangle$. Our main results are presented in Fig. 2 . We used $2 \times 2$ scattering matrices parametrized by $\hat{s}_{2}^{-1} \hat{s}_{1}=\exp \left(i \phi \sigma_{x}\right)$ and repeated the calculation for several $\phi \in[0, \pi]$. Small $\phi$

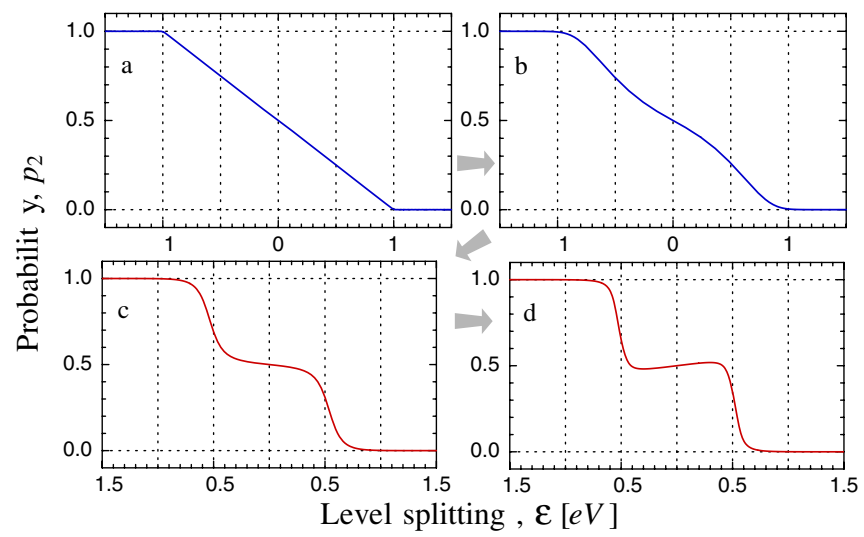

FIG. 2 (color online). The probability $p_{2}$ that the qubit is in state $|2\rangle$ vs level splitting $\varepsilon$. At weak coupling between the QPC and qubit, (a),(b) the transfer of a single electron gives rise to cusps in $p_{2}$ at $\pm e V$. Peculiarities at $\pm e V / 2$ (c),(d) dominate the signal at strong coupling. Scattering matrices were parametrized as stated in the text. (a),(b),(c),(d) respectively, correspond to $\phi=\pi / 16, \pi / 4,7 \pi / 10$, and $4 \pi / 5$. corresponds to weak coupling. The curve at $\phi=\pi / 16$ is almost indistinguishable from the perturbative weak coupling limit discussed in the introduction. Cusps at $\pm \mathrm{eV}$ indicate that qubit switching is accompanied by the transfer of single electrons in the QPC.

The increasing decoherence smooths the cusps for the curve at $\phi=\pi / 4$ (2b). When the coupling is increased beyond $\phi=\pi / 2$ steps appear at $\pm e V / 2$ (c). Further increase of the coupling results in a sharpening of the steps (d).

Let us now briefly consider the limit of strong coupling where the qubit significantly affects the scattering in many QPC-channels. In this case, $\tilde{P}(\varepsilon)$ is approximately a Gaussian, $\tilde{P}(\varepsilon) \propto \exp \left(-\frac{\varepsilon^{2}}{2 \beta(\mathrm{eV})^{2}}\right)$ with $\beta$ a large dimensionless number proportional to the number of channels. The interpretation of this is that electron fluctuations in the QPC affect the qubit level splitting. The typical fluctuation induced is $\delta \varepsilon \sim e V \sqrt{\beta}$. The frequency scale of the fluctuations is $e V$ which is small compared to $\delta \varepsilon$. The fluctuations are, therefore, quasistationary. Their distribution are Gaussian by virtue of the central limit theorem. This leads to a pseudothermal polarization $p_{2}=1 /\left(1+\exp \left(\varepsilon / k_{B} T^{*}\right)\right.$ where the effective temperature $k_{B} T^{*}=2 \sqrt{\beta / \alpha} \mathrm{eV}$ is of the order of $e V$. The constant $\beta$ is evaluated from numerics. For example, for $N \gg 1$ identical channels with scattering matrices $\exp \left(i \phi \sigma_{x}\right)$ and $\phi=3 \pi / 4$ we find $\beta \approx$ $N / 7$ and effective temperature $\approx 0.36 \mathrm{eV}$. The added decoherence inherent in a many-channel QPC smooths out all peculiarities. Details of the calculation are presented in the supplementary material [19].

Let us speculate about the origin of the $\varepsilon=e V / 2$ peculiarities. It would have been easy to explain peculiarities at $\varepsilon=n e V, n=2,3,4, \ldots$ in $p_{2}(\varepsilon)$ as resulting from the transfer of multiple electrons. But for fractional peculiarities we have to turn to an indirect analogy with the model of interacting particles on a ring threaded by a magnetic flux [20]. There, one expects that the energy eigenvalues are periodic in flux with period of one flux quantum. However, the exact Bethe-ansatz solution [20] reveals a double period of eigenvalues with adiabatically varying flux.

For our nonequilibrium setup, energy eigenvalues are not particularly useful. The natural eigenvalues to describe the phenomenon are those of the operator $\tilde{Q}^{(V)}(\tau)$. They depend on the parameter $e V \tau$ which is an analogue of flux. The product of the eigenvalues, i.e., the determinant $\tilde{P}(\tau)$ is not precisely periodic in $\tau$ since it decays at large $\tau$ owing to decoherence. Still, it oscillates and the period of these oscillations doubles as we go from weak to strong coupling [Fig. 3(b)]. The doubling can be understood in terms of the transfer of the eigenvalues of $\tilde{Q}^{(V)}(\tau)$ upon increasing $\tau$ [Fig. 3(a)] assuming the parametrization $\hat{s}_{2}^{-1} \hat{s}_{1}=$ $\exp \left(i \phi \sigma_{x}\right)$ : In the large $\tau$ limit, energy-time uncertainty can be neglected in a "quasiclassical" approximation: The operator $\hat{\Pi}(\tau)$ projects onto a very long time interval, and 


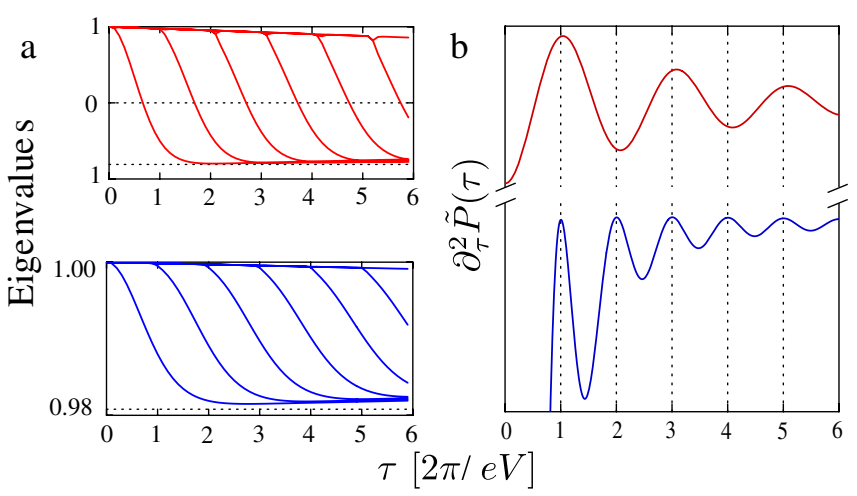

FIG. 3 (color online). The behavior of eigenvalues (a) and the determinant $\tilde{P}(\tau)$ (b) at weak and strong QPC-qubit coupling, respectively. The parameter $\phi$ in equals $4 \pi / 5$ (top) and $\pi / 16$ (bottom) representing the strong and weak coupling limits, respectively. Deviations from the correct asymptotics are due to finite size effects. (b) contains the second derivative of $\tilde{P}(\tau)=$ $\operatorname{det} \hat{Q}^{(0)}(\tau)^{-1} \hat{Q}^{(V)}(\tau)$. (The second derivative is taken to remove an average slope and curvature.)

is replaced by the identity operator. $\tilde{Q}^{(V)}$ becomes diagonal in energy. All eigenvalues that are not equal to 1 are concentrated in the transport energy window $0<E<$ $e V$, where the filling factors in the QPC reservoirs are not the same. For $\hat{s}_{2}^{-1} \hat{s}_{1}$ parametrized as above, these eigenvalues equal $\cos (\phi)$. There are $e V \tau / 2 \pi$ of them. In other words, the number of eigenvalues equal to $\cos \phi$ grows linearly with $\tau$. Numerical diagonalization of $\tilde{Q}^{(V)}(\tau)$ [Fig. 3(a)] shows that one eigenvalue is transferred from 1 to $\cos (\phi)$ during time $2 \pi / e V$. If $\cos (\phi)>0$ as in the weak coupling case [bottom of Fig. 3(b)], this gives rise to $P(\tau)$ oscillations with frequency $e V / 2 \pi$ manifesting integer charges. However, $\cos \phi$ becomes negative at stronger couplings, so that $P(\tau)$ changes sign with each eigenvalue transfer [Fig. 3(b) (top)]. Two eigenvalues have to transfer to give the same sign. The result is a period doubling of the oscillations in $\tilde{P}(\tau)$. This resembles the behavior of the wave vectors of the Bethe-Ansatz solution in [20].

The parametrization of the $\hat{s}_{2}^{\dagger} \hat{s}_{1}=\exp \left(i \phi \sigma_{x}\right)$ is not general. However, the eigenvalue transfer arguments help to understand general scattering matrices. Eigenvalue transfer still occurs at frequency $\mathrm{eV} / 2 \pi$ but instead of traveling along the real line, eigenvalues follow a trajectory inside the unit circle in the complex plane. Peculiarities at fractional level splittings $e V / 2$ are pronounced if the end point of the trajectory has a negative real part. Numerical results for general scattering matrices are presented in the supplementary material [19].

Results presented so far are for "spinless" electrons. Spin degeneracy is removed by, e.g., high magnetic field. If spin is included, but scattering remains spin independent, then two degenerate eigenvalues are transported simulta- neously. In this case, the $\mathrm{eV} / 2$ peculiarities disappear for the parametrization $\exp \left(i \phi \sigma_{x}\right)$ but persists for more general scattering matrices. The results of further numerical work that confirm this are presented in supplementary material [19].

We have studied a quantum transport setup that can easily be realized with current technology, namely, that of a quantum point contact coupled to a charge qubit. The qubit is operated as a measuring device, its output signalthe polarization $p_{2}$-is directly seen in the QPC current. When the qubit is weakly coupled to the QPC, the dependence is dominated by processes where a single QPC electron interacts with the qubit. For intermediate couplings, the dependence shows peculiarities at level splittings $\pm e V / 2$. These peculiarities are the result of manybody correlations induced in the QPC by qubit switching. Decoherence destroys these peculiarities in the limit where the qubit couples many QPC channels, leading to a pseudo Boltzmann polarization with effective temperature $e V$.

[1] B. J. Van Wees et al., Phys. Rev. Lett. 60, 848 (1988).

[2] M. Büttiker, Phys. Rev. B 41, 7906 (1990).

[3] L. S. Levitov, H. Lee, and G. H. Lesovik, J. Math. Phys. (N.Y.) 37, 4845 (1996).

[4] C. W. J. Beenakker, C. Emary, and M. Kindermann, Phys. Rev. Lett. 91, 147901 (2003).

[5] J. M. Elzerman et al., Phys. Rev. B 67, 161308(R) (2003).

[6] J. R. Petta et al., Phys. Rev. Lett. 93, 186802 (2004).

[7] I. L. Aleiner, N. S. Wingreen, and Y. Meir, Phys. Rev. Lett. 79, 3740 (1997).

[8] Y. Levinson, Europhys. Lett. 39, 299 (1997).

[9] E. Onac et al., Phys. Rev. Lett. 96, 176601 (2006).

[10] J. Tobiska, J. Danon, I. Snyman, and Y. V. Nazarov, Phys. Rev. Lett. 96, 096801 (2006).

[11] Y. V. Nazarov and M. Kindermann, Eur. Phys. J. B 35, 413 (2003).

[12] D. A. Abanin and L. S. Levitov, Phys. Rev. Lett. 93, 126802 (2004).

[13] D. A. Abanin and L. S. Levitov, Phys. Rev. Lett. 94, 186803 (2005).

[14] P. W. Anderson, Phys. Rev. Lett. 18, 1049 (1967).

[15] G. D. Mahan, Phys. Rev. 163, 612 (1967).

[16] P. Nozières and C. T. De Dominicic, Phys. Rev. 178, 1097 (1969).

[17] K. A. Matveev and A. I. Larkin, Phys. Rev. B 46, 15337 (1992).

[18] K. Yamada and K. Yosida, Prog. Theor. Phys. 68, 1504 (1982).

[19] See EPAPS Document No. E-PRLTAO-99-022736 for additional numerical results and details of the numerical calculation. For more information on EPAPS, see http:// www.aip.org/pubservs/epaps.html.

[20] B. Sutherland and B. S. Shastry, Phys. Rev. Lett. 65, 1833 (1990). 